\title{
Co-Registered Spectrally Encoded Confocal Microscopy and Optical Frequency Domain Imaging System
}

\section{Citation}

Kang DK, Suter MJ, Boudoux C, Yachimski PS, Puricelli WP, Nishioka NS, Mino-Kenudson M, Lauwers GY, Bouma BE, and Tearney GJ. Co-registered spectrally encoded confocal microscopy and optical frequency domain imaging system. Journal of Microscopy 239, no. 2: 87-91.

doi:10.1111/j.1365-2818.2010.03367.x

\section{Published Version}

doi:10.1111/j.1365-2818.2010.03367.x

\section{Permanent link}

http://nrs.harvard.edu/urn-3:HUL.InstRepos:32539237

\section{Terms of Use}

This article was downloaded from Harvard University's DASH repository, and is made available under the terms and conditions applicable to Other Posted Material, as set forth at http:// nrs.harvard.edu/urn-3:HUL.InstRepos:dash.current.terms-of-use\#LAA

\section{Share Your Story}

The Harvard community has made this article openly available.

Please share how this access benefits you. Submit a story.

Accessibility 


\title{
Co-registered spectrally encoded confocal microscopy and optical frequency domain imaging system
}

\author{
D.K. Kang ${ }^{\star}$, M.J. Suter ${ }^{\star}$, C. Boudoux ${ }^{\star}$, P.S. Yachimski ${ }^{\dagger}$, W.P. Puricelli ${ }^{\dagger}$, N.S. Nishioka ${ }^{\star} \dagger$, M. \\ Mino-Kenudson ${ }^{\ddagger}$, G.Y. Lauwers ${ }^{\ddagger}$, B.E. Bouma ${ }^{*}$, and G.J. Tearney ${ }^{\star}, \ddagger$ \\ *Wellman Center for Photomedicine, Massachusetts General Hospital and Harvard Medical \\ School, Boston 02114, MA, U.S.A. \\ ${ }^{\dagger}$ Gastrointestinal Unit, Massachusetts General Hospital and Harvard Medical School, Boston \\ 02114, MA, U.S.A. \\ ¥Pathology Service, Massachusetts General Hospital and Harvard Medical School, Boston \\ 02114, MA, U.S.A.
}

\section{Summary}

Spectrally encoded confocal microscopy and optical frequency domain imaging are two noncontact optical imaging technologies that provide images of tissue cellular and architectural morphology, which are both used for histopathological diagnosis. Although spectrally encoded confocal microscopy has better transverse resolution than optical frequency domain imaging, optical frequency domain imaging can penetrate deeper into tissues, which potentially enables the visualization of different morphologic features. We have developed a co-registered spectrally encoded confocal microscopy and optical frequency domain imaging system and have obtained preliminary images from human oesophageal biopsy samples to compare the capabilities of these imaging techniques for diagnosing oesophageal pathology.

\section{Keywords}

Confocal microscopy; co-registered imaging; multi-modality imaging; optical coherence tomography

\section{Introduction}

In vivo microscopic optical imaging methods hold considerable promise for non-excisional histopathological diagnosis. Two techniques, reflectance confocal microscopy (RCM) and optical coherence tomography (OCT) are particularly well suited for non-invasive microscopy in patients as they are capable of allowing the visualization of microscopic structure without tissue contact and do not require the administration of exogenous contrast agents. OCT and RCM reject or ignore multiply scattered light from tissue and detect the singly backscattered photons that contain microstructural information. Each technique rejects multiply scattered light in a different way: OCT utilizes coherence gating and RCM employs confocal selection of light reflected from tissue illuminated by a tightly focused beam. OCT provides cross-sectional images with typical resolutions of $30(\mathrm{H}) \times 30(\mathrm{~W}) \times$ $10 \mu \mathrm{m}(\mathrm{D})$ and a penetration depth that ranges from 1 to $3 \mathrm{~mm}$, depending on tissue type. 
RCM obtains transverse or en face images with typical resolutions of $1(\mathrm{H}) \times 1(\mathrm{~W}) \times 6 \mu \mathrm{m}$ (D) and a penetration depth that ranges from 100 to $300 \mu \mathrm{m}$. Based on these features of the two imaging modalities, it is generally accepted that they offer different information: RCM provides transverse subcellular detail and OCT provides cross-sectional architectural morphology; information from these two resolution regimes is critical for histopathological diagnosis.

Recently developed RCM technology termed spectrally encoded confocal microscopy(SECM)and a second-generation OCT technology called optical frequency domain imaging (OFDI - also known as swept source OCT) have the advantages that they can be utilized to conduct high speed imaging over large fields endoscopically. SECM is a wavelength-division multiplexed confocal approach that uses a broadband or wavelengthswept light source and a diffraction grating to encode one dimension of spatial information in the optical spectrum (Tearney et al., 1998; Boudoux et al., 2005). OFDI utilizes a rapid wavelength-swept laser to generate spectral interference that, once detected, is Fourier transformed to form an image (Choma et al., 2003; Yun et al., 2003). In this paper, we describe the development of a multi-modal imaging system that creates co-registered threedimensional images for each of SECM and OFDI imaging modalities. Preliminary images of human oesophageal biopsy samples were obtained by the imaging system to evaluate capabilities of these imaging techniques for diagnosing oesophageal pathology.

\section{Methods}

A schematic image of the co-registered SECM and OFDI system is illustrated in Fig. 1.For SECM, light from a wavelength-swept source (centre wavelength $=1320 \mathrm{~nm}$; bandwidth $=$ $70 \mathrm{~nm}$; instantaneous linewidth $<0.1 \mathrm{~nm}$; repetition rate $=5 \mathrm{kHz}$ ) (Boudoux et al., 2005) was transmitted through a 50/50 beam splitter and diffracted by a holographic transmission grating (groove density $=1100$ lines $/ \mathrm{mm}$ ). The diffracted light was relayed by telescope optics (magnification $=2.4$ ) onto an objective lens [numerical aperture $(\mathrm{NA})=0.9 \mathrm{w} ; f=5.3 \mathrm{~mm}$ ]. In order to simulate the NA that we may be able to achieve in a centred oesophageal probe, the aperture of the objective lens was under-filled to achieve an effective NA of 0.7. The power on the sample was measured to be $5.4 \mathrm{~mW}$. The reflected light from the sample returned back to the beam splitter, where it was reflected towards a detection fibre. In order to reduce laser speckle in the SECM image, the core of a multi-mode fibre (core diameter $=62.5 \mu \mathrm{m}$ ) was employed as the detection aperture (Boudoux, 2007). The image of the detection aperture at the sample plane had a diameter of $5.5 \mu \mathrm{m}$. The electric signal from the detector was sampled at a rate of $5 \mathrm{Ms} \mathrm{s}^{-1}$, with a 12-bit depth, and 700 pixels were digitized for each line. The objective lens was scanned along the axial direction by a piezoelectric transducer actuator to obtain confocal optical sections at different depths in the tissue.

An additional wavelength-swept source (centre wavelength $=1320 \mathrm{~nm}$; bandwidth $=143$ $\mathrm{nm}$; instantaneous linewidth $=0.16 \mathrm{~nm}$; repetition rate $=40 \mathrm{kHz}$ ) was used for the OFDI system, which has been described in detail in prior publications (Yun et al., 2003, 2004, 2006). One dimension of the image was obtained by using a mirror mounted on a galvanometer, which scanned the sample arm beam. Relay optics (magnification $=0.5$ ) was used to project the aperture of the scanner onto the back focal aperture of the objective lens and collimate the beam entering the objective lens. The back focal aperture was under-filled to provide an effective NA of 0.064 . The power on the sample was measured to be $54 \mathrm{~mW}$. The system utilized two detectors for polarization diversity; each detected signal was digitized at a rate of $80 \mathrm{Ms} \mathrm{s}^{-1}$, with a 14-bit depth. There were 2048 samples per A-line for each detector channel. 
SECM and OFDI modalities were selected by using a switchable fold mirror. The fold mirror was switched out when SECM was used and switched in when OFDI was in use. The same objective lens was used for both of the imaging modalities, which made it possible to precisely co-register SECM and OFDI images. Two perpendicular translational stages were used to scan a large area on the sample.

In order to demonstrate the co-registered imaging system, biopsy samples taken from patients undergoing routine oesophagogastroduodenoscopy were imaged fresh, immediately after removal from the patient. The biopsy samples were imaged through a BK7 cover slip and immersed in phosphate buffered saline. For SECM, entire biopsy samples were imaged at 15 different focal planes spaced by $10 \mu \mathrm{m}$. The same regions were imaged by OFDI. Following imaging, the samples were processed for routine paraffin-embedded histology, sectioned and stained with haematoxylin and eosin. The study protocol was approved by the Partners Internal Review Board (IRB \#2007P000656). A total of 14 biopsy samples have been imaged to date.

\section{Results and discussion}

The transverse resolution of the multimodality system was measured by imaging a 1951 USAF resolution target (Fig. 2). For SECM, the full width-half-maximum (FWHM) of the line spread function were measured to be $2.3 \pm 0.12 \mu \mathrm{m}$ and $1.8 \pm 0.15 \mu \mathrm{m}$ along the spectrally encoded direction and the direction perpendicular to the spectrally encoded line, respectively. The transverse line spread FWHM was larger along the spectrally encoded direction due to the finite width of the instantaneous spectrum of the wavelength-swept source and the limited bandwidth of the photodetector. The transverse resolution of OFDI (FWHM of the line spread function) was measured to be $11.4 \pm 1.5 \mu \mathrm{m}$. The axial resolution of SECM was measured by scanning a mirror along the axial direction. The measured FWHM of the axial plane response function was $9.7 \pm 0.44 \mu \mathrm{m}$. For OFDI, the axial plane response, measured using an A-scan of a mirror, was $8.4 \pm 0.1 \mu \mathrm{m}$ in air.

Figure 3 shows one of the data sets from this biopsy study. For this case, the biopsy sample was taken from a patient with the history of Barrett's oesophagus with high-grade dysplasia and treatment with photodynamic therapy. Low-magnification en face images of SECM (Fig. 3a) and OFDI (Fig. 3b) distinguish gastric cardia-type mucosa (G) from normal squamous mucosa (S). Registration error between the SECM and OFDI en face images was measured by calculating the location of the maximum of the two-dimensional crosscorrelation of the two images. Registration error was measured to be 50 and $65 \mu \mathrm{m}$ or $1.0 \%$ and $2.3 \%$ of the field of view along the $x$ - and $y$-axes, respectively. When the images were compensated post hoc for the registration error (Fig. 3a and b), they showed good spatial correspondence. The magnified SECM image (Fig. 3c) of the junctional region marked by a white box in Fig. 3(a) demonstrates squamous cells with occasionally visible nuclei (arrowheads) from columnar cells (solid arrows), with cellular features that are similar to those shown in high-magnification histopathology image (Fig. 3h), whereas these features are not as well appreciated by the corresponding OFDI image (Fig. 3d).

Low-magnification cross-sectional images of SECM (Fig. 3e) and OFDI (Fig. 3f), obtained along the dotted lines in Fig. 3(a) and (b), respectively, also showed differences between gastric cardia-type mucosa $(\mathrm{G})$ and normal squamous mucosa (S), including the presence of gastric pits. Unlike the en face SECM image, cellular details were not well observed in the cross-sectional SECM image due to its poorer resolution along the axial direction than the transverse direction. We set the maximum number of SECM axial scans as 15, which kept the imaging time less than $20 \mathrm{~min}$, which was required to preserve the sample for subsequent histological processing. As a result, the ranging depth for SECM was limited to 
$150 \mu \mathrm{m}$ making a thorough comparison of cross-sectional images difficult. Haematoxylin and eosin stained histopathology (Fig. $3 \mathrm{~g}$ and $\mathrm{h}$ ) confirmed that the sample is composed of squamous mucosa $(\sim 25 \%)$ and cardia-type mucosa $(\sim 75 \%)$.

In this paper, we have demonstrated a co-registered SECM and OFDI system capable of providing images with two different resolution scales and display formats. Preliminary images demonstrated good spatial correspondence between the two imaging modalities. Although both SECM and OFDI visualized architectural features of oesophageal biopsy samples, cellular features were better appreciated in SECM than in OFDI. Whether these additional cellular features visualized in SECM provide better diagnostic accuracy than OFDI needs further study, which can be effectively conducted by the co-registered imaging system.

We have furthermore learned that co-registered images provide us with an opportunity to verify and possibly understand OFDI diagnostic criteria better. For instance, one of the diagnostic criteria for gastric cardia (which is important to distinguish from Barrett's oesophagus, also commonly termed specialized intestinal metaplasia) is a highly reflective line at the mucosal surface (red dashed arrows in Fig. 3b, d and f) (Evans et al., 2006, 2007). This highly reflective signal was also observed at the apical aspect of cells in SECM (red dashed arrows in Fig. 3a, c and e), confirming that the surface of cardia epithelial cells is indeed scattering differently and that this effect is not an artefact seen on OFDI. The highmagnification SECM en face image (Fig. 3c) revealed that the origin of this strong reflection (red dashed arrow) is not cell nuclei (white arrows) - information that might be difficult to extract from the OFDI image alone. We anticipate that we will be able to confirm and/or verify other features commonly seen by OFDI in a similar manner.

In this study, it was difficult to achieve high degree of spatial correspondence between the images obtained by the multimodality system and the histopathology images. This registration inaccuracy was primarily caused by loss of tissue orientation during the fixation and sectioning process for histopathology. Better registration can be achieved by threedimensional histology of the biopsy specimen (Boag et al., 2001). The three-dimensional data set can then be re-sliced digitally to generate two-dimensional histopathology images that have a similar orientation and location to the SECM and OFDI images.

Similar to the OFDI probe, comprehensive imaging of distal oesophagus will be conducted by helically scanning the SECM probe through a balloon-centring catheter (Suter et al., 2008). We have previously demonstrated high-speed microscopic imaging over large luminal areas with a bench-top SECM probe (Yelin et al., 2007). Remaining challenges include integrating an axial scanning mechanism for the objective lens and conducting adaptive focusing to compensate for the patient movement and tissue surface irregularities. Another SECM probe that utilizes active feedback derived from an optical signal emanating from the tissue surface and a miniature piezoelectric transducer actuator to control the focal location is presently under development.

Finally, the accuracy of SECM for diagnosis of relevant oesophageal pathology is unknown. We intend to use the device described here in a larger biopsy study to compare the diagnostic accuracy of each modality. This information will be critical for determining the next appropriate steps for the development of this technology.

\section{Acknowledgments}

This research is supported by National Cancer Institute (grant number R21CA122161). Caroline Boudoux is currently with École Polytechnique Montréal, Canada, and Patrick S. Yachimski is with Vanderbilt University Medical Center, Nashville, Tennessee, U.S.A. 


\section{References}

Boag AH, Kennedy LA, Miller MJ. Three-dimensional microscopic image reconstruction of prostatic adenocarcinoma. Arch. Pathol. Lab. Med. 2001; 125:562-566. [PubMed: 11260639]

Boudoux, C. Wavelength Swept Spectrally Encoded Confocal Microscopy for Biological and Clinical Applications. Cambridge, MA: Division of Health Sciences and Technology and Department of Nuclear Science and Engineering, Massachusetts Institute of Technology; 2007.

Boudoux C, Yun S, Oh W, et al. Rapid wavelength-swept spectrally encoded confocal microscopy. Opt. Express. 2005; 13:8214-8221. [PubMed: 19498851]

Choma M, Sarunic M, Yang C, Izatt J. Sensitivity advantage of swept source and Fourier domain optical coherence tomography. Opt. Express. 2003; 11:2183-2189. [PubMed: 19466106]

Evans JA, Poneros JM, Bouma BE, et al. Optical coherence tomography to identify intramucosal carcinoma and high-grade dysplasia in Barrett's esophagus. Clin. Gastroenterol. Hepatol. 2006; 4:38-43. [PubMed: 16431303]

Evans JA, Bouma BE, Bressner J, et al. Identifying intestinal metaplasia at the squamocolumnar junction by using optical coherence tomography. Gastrointest. Endosc. 2007; 65:50-56. [PubMed: 17137858]

Suter MJ, Vakoc BJ, Yachimski PS, Shishkov M, Lauwers GY, Mino-Kenudson M, Bouma BE, Nishioka NS, Tearney GJ. Comprehensive microscopy of the esophagus in human patients with optical frequency domain imaging. Gastrointest. Endosc. 2008; 68:745-753. [PubMed: 18926183]

Tearney GJ, Webb RH, Bouma BE. Spectrally encoded confocal microscopy. Opt. Lett. 1998; 23:1152-1154. [PubMed: 18087457]

Yelin D, Boudoux C, Bouma BE, Tearney GJ. Large area confocal microscopy. Opt. Lett. 2007; 32:1102-1104. [PubMed: 17410249]

Yun S, Tearney G, de Boer J, Iftimia N, Bouma B. High-speed optical frequency-domain imaging. Opt. Express. 2003; 11:2953-2963. [PubMed: 19471415]

Yun S, Tearney G, de Boer J, Bouma B. Removing the depth-degeneracy in optical frequency domain imaging with frequency shifting. Opt. Express. 2004; 12:4822-4828. [PubMed: 19484034]

Yun SH, Tearney GJ, Vakoc BJ, et al. Comprehensive volumetric optical microscopy in vivo. Nat. Med. 2006; 12:1429-1433. [PubMed: 17115049] 


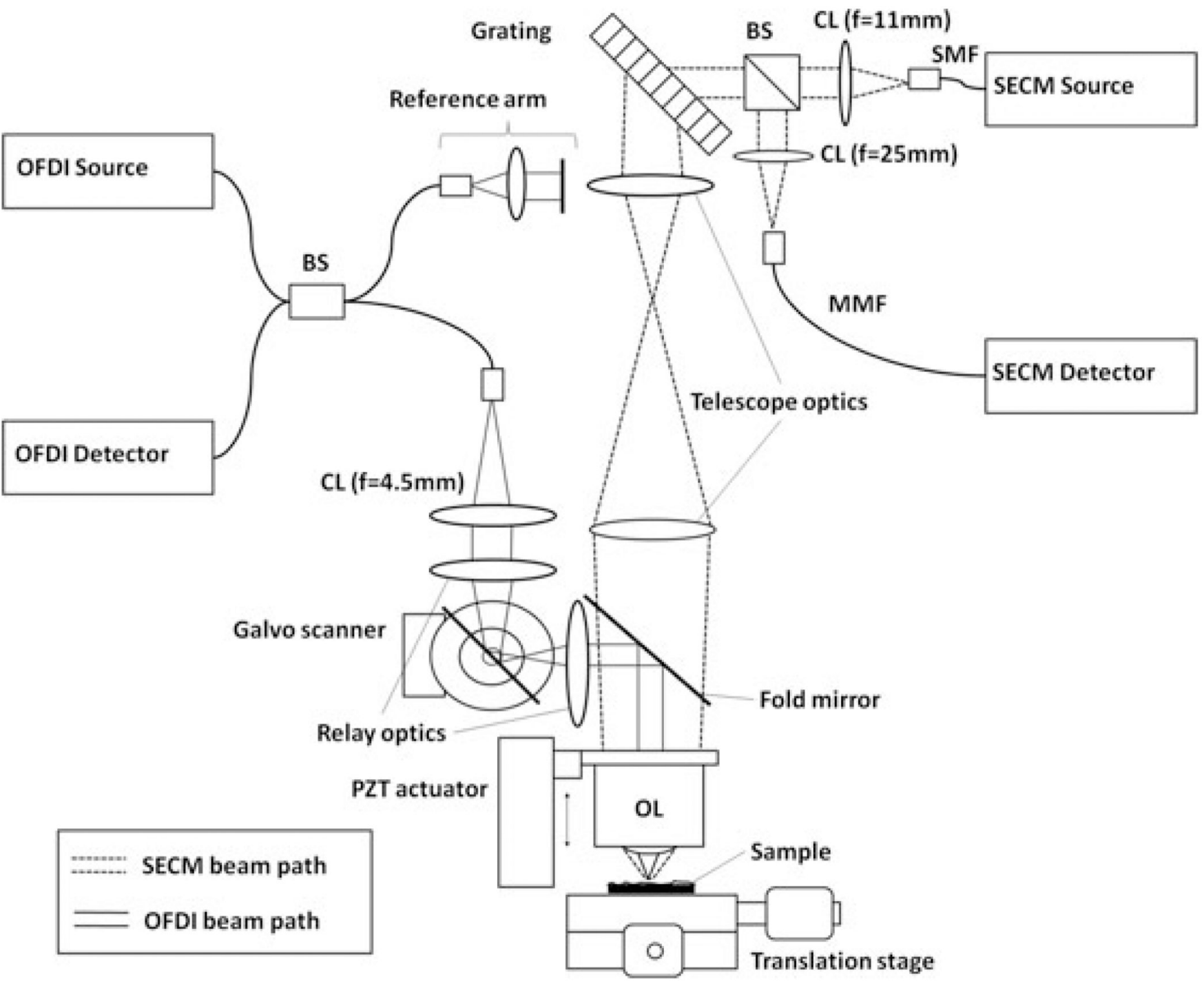

Fig. 1.

Schematic of co-registered SECM and OFDI system. BS, beam splitter; CL, collimation lens; SMF, single-mode fibre; MMF, multi-mode fibre; OL, objective lens; PZT, piezoelectric transducer. 


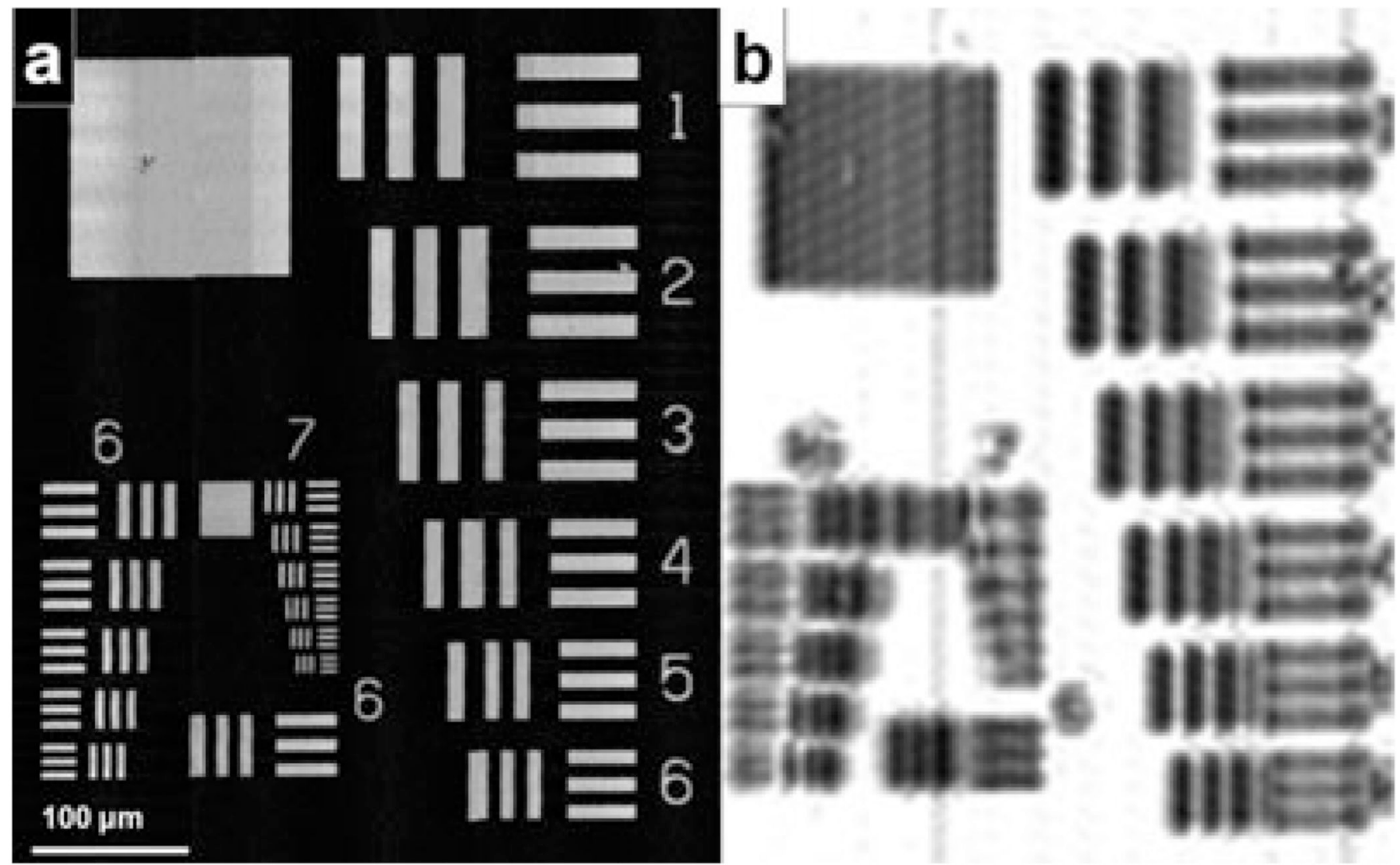

Fig. 2.

Images of a 1951 USAF resolution target obtained by SECM (a) and OFDI (b). 

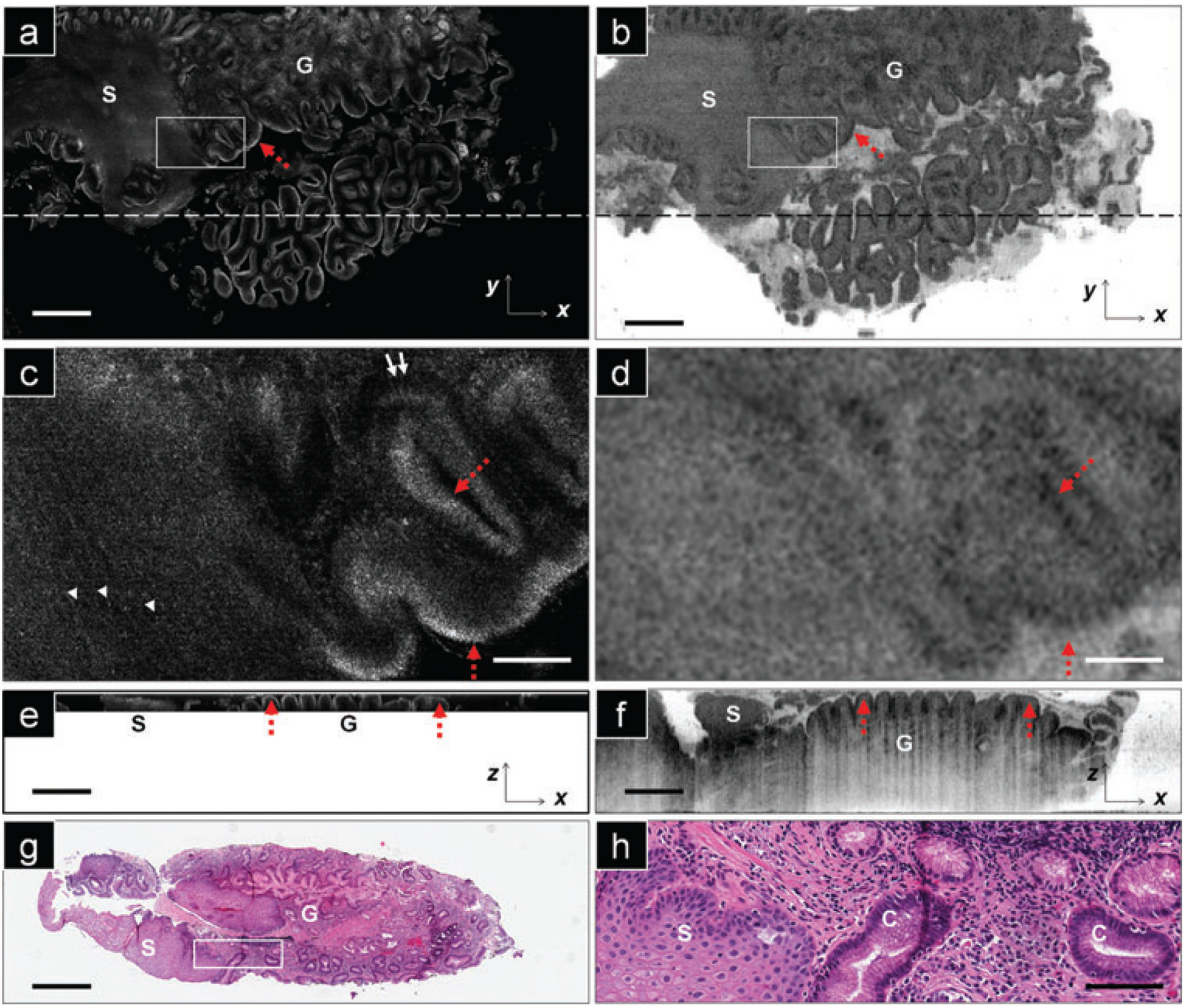

Fig. 3.

Images of human oesophageal biopsy sample showing the gastroesophageal junction. Lowmagnification en face images of SECM (a) and OFDI (b). High-magnification en face images of SECM (c) and OFDI (d). Low-magnification cross-sectional images of SECM (e) and OFDI (f). Haematoxylin and eosin stained histology in low-(g) and high-(h) magnifications. S, squamous mucosa; G, gastric cardia-type mucosa; C, columnar cells; red dashed arrow, highly reflecting surface of cardia-type mucosa; arrowhead, nuclei in squamous cells; solid arrow, columnar cells. Scale bars in (a), (b), (e), (f) and (g) represent $500 \mu \mathrm{m}$. Scale bars in (c), (d) and (h) represent $100 \mu \mathrm{m}$. 